\title{
SYSTEMS OF ELECTRONIC OVERCURRENT PROTECTION IN PULSE POWER GENERATOR OPERATING ON PLASMA LOAD
}

\author{
Dmitriy V. Godun, Sergey V. Bordusov*, Anatoliy P. Dostanko \\ Belorussian state university of informatics and radioelectronics, Minsk, Belarus \\ * corresponding author: bordusov@bsuir. by
}

\begin{abstract}
Schematic peculiarities of pulsed power source and modulator-shaper for working on high instability plasma load are discussed. In its construction should be provided for several levels of overcurrent protection. First of all modules of electronic protection should be integrated into the control driver system of IGBT modules and must provide a quick disconnect power switches in excess of the allowable values of pulse current. The next level of overcurrent protection in pulse power generator is a protection against overcurrent in the load circuit. Operating threshold of current protection in this case must be set to the maximum value of current in the secondary circuit. In order to limit the emission of stray voltage on the power pulses in a moment of switching of power switches a restrictive $\mathrm{RC}$ snubbers parallel to the collectors and the emitters of transistors must be installed. It is also appropriate application of software-controlled configurating of electrical power at the output of a pulsed power supply.
\end{abstract}

KEYWORDS: plasma, high-voltage source, pulse voltage.

\section{INTRODUCTION}

The electronic system of complex protection of pulse power generator is used for the protection of key IGBT transistors against excess of permissible pulse current values and temperature during gas discharge formation at a low pressure. An application peculiarity of the pulse power generator during the gas discharge formation is a wide range of setting of operating parameters and consequently instability of plasma as the load [3]. The fast-acting complex protection system of pulse power generator is to ensure its blocking with the lapse of time not exceeding 100 nanoseconds.

\section{MAIN PART}

The complex electronic protection system of pulse power generator is shown in Fig. 1.

The pulse generator [1, operating into plasma load, composes four current protection circuits and two thermal protection circuits of power semiconductor devices. The system has been designed in such a way that thermal and current protections are interconnected and operate simultaneously. Their joint operation ensures a quick switch-off of the power generator in case of excess of the permissible pulse current values or temperature of power semiconductor devices.

The thermal protection circuit is required to control the temperature of IGBT transistors operating in a pulse mode. According to the producer's technical documentation with the increase of the temperature of IGBT transistors up to $100^{\circ} \mathrm{C}$ the permissible pulse current of the device decreases by two times [5]. In this case to determine a value of a threshold of electrical current pulse limit depending on temperatures of transistor circuits is a task of current importance.

The electronic protection system is divided into several circuits. The main circuit of electronic protection (the first circuit) has been implemented in the composition of the driver control system of IGBT transistors (INVERTOR). The electronic protection system includes sensors of response temperature at $70^{\circ} \mathrm{C}$. According to the operation algorithm of the electronic protection system, when the response temperature is reached the power transforming cascades are switched off. The cogitative control system constantly monitors the temperature with digital sensors. The data are processed by the microcontroller and, in accordance with the computed temperature, the threshold of current protection system actuation is corrected with the help of digital potentiometers [2]. The system operates in a phased control mode and ensures a stable dynamic correlation between a value of pulse current flowing through the key element and the temperature of its frame. In the circuit the current protection module as a part of the invertor is implemented on a fast-acting comparator to which input comes a signal from the current sensor RS1. This signal is compared with the basic signal and if they are equal the comparator is switched over. The trigger $\mathrm{T}$ has been introduced into the electronic protection circuit to exclude transients at the moment of comparator's switching and keep the system's last logic status. The trigger is reset either at a signal of the microcontroller or when the power of the generator is turned off. When logic-1 level is input to SD microcircuit of the bridge converter driver the generator is 


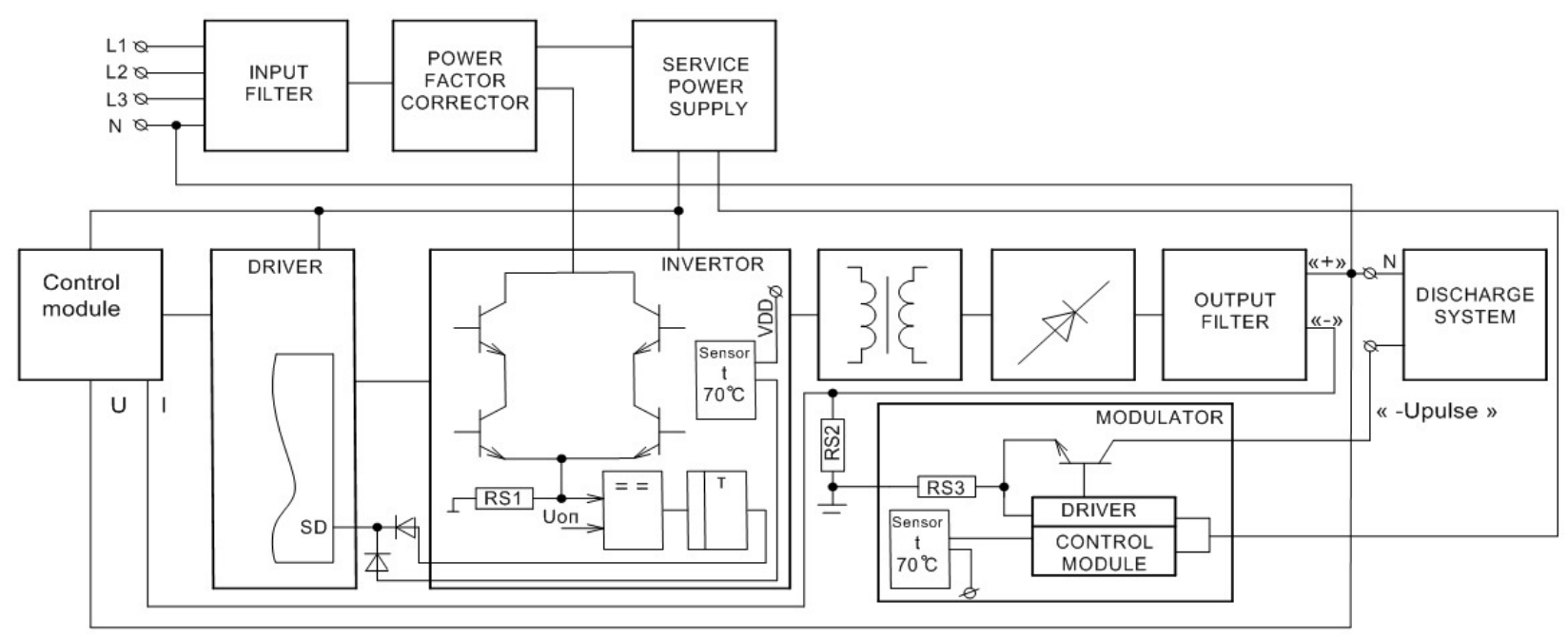

FiguRE 1. Structure chart of the comlex protection system of pulse generator.

rapidly turned off. This signal is formed by the modules of current and temperature protections when the preset limits of pulse current and temperature values of IGBT transistors are reached.

The aim of the second circuit of current protection of pulse power supply is to determine the value of current in the load circuit (DISCHARGE SYSTEM) and, in case it goes beyond the permissible limit, to instantly turn off the generator. The threshold of protection response in the second circuit is set $10 \div 15 \%$ higher of the threshold of protection response in the third circuit and is set to the maximum permissible value of current in the load circuit (DISCHARGE SYSTEM).

The third circuit is software operated and allows to maintain the level of the preset power at the output of the generator. Its operation is based in such a way that a signal from the current sensor RS2 comes to the special input of pulse generator's control system forming a circuit of a negative feedback on current. The plasma discharge, as the load, is especially instable and lets a short-term excess of the pulse current when the output parameters of the pulse generator are changed. If the excess of the permissible level of pulse current in the load (DISCHARGE SYSTEM) is achieved by force of the software then the amplitudes of power pulses are decreased and the power transmitted to the discharge is stabilized. When the value of the pulse current in the load (DISCHARGE SYSTEM) is lowered to the permissible limits then the amplitude of the power pulses is restored.

When elaborating current protection systems optical systems of data transfer with the switch period up to 75 ns has been applied which ensure the retention interval necessary to turn the generator off.

To get improved frequency characteristics the modulator's key power cascade is made of several MOSFET transistors switched sequentially (Fig. 2). The application of MOSFET transistors is preferable because

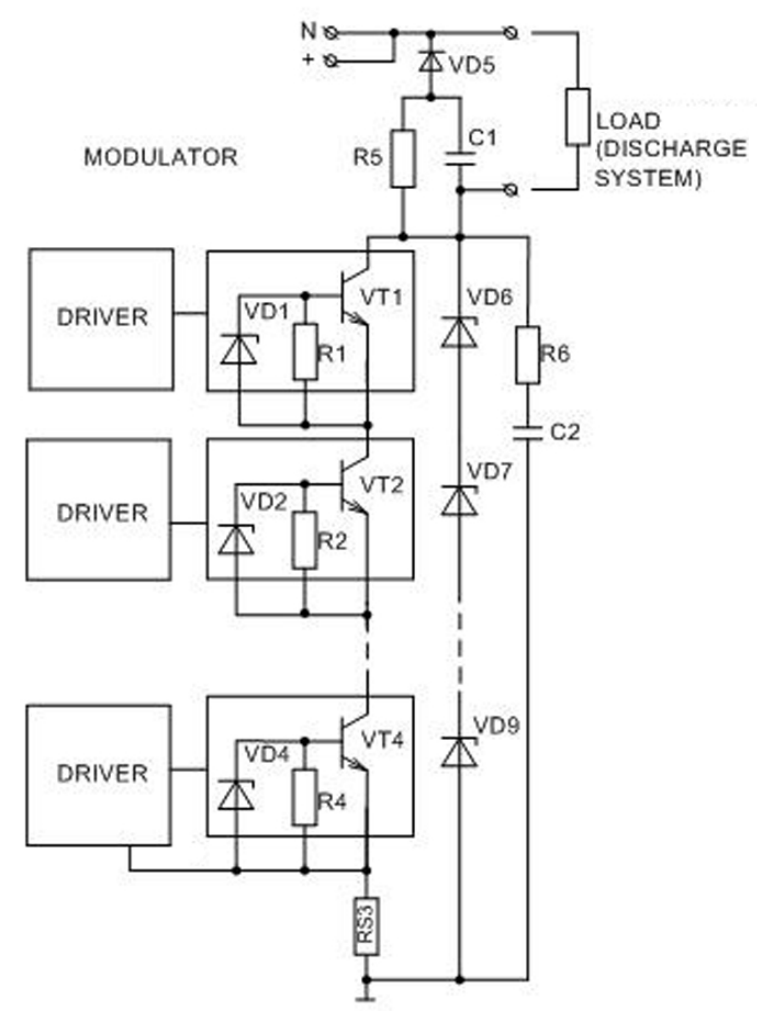

FiguRE 2. Structure chart of the modulator.

of a rather high switching frequency of power voltage with a relatively small gate capacitance which ensures the formation of high trajectories switching [4]. Each transistor is controlled by a personal driver device. The driver of the lower transistor includes current protection device which limits the pulse current in the load (DISCHARGE SYSTEM) and when the preset limit of the current value is reached it turns off the system of control signals formation. The transistors must be safely closed and the power circuit 
must be open. In order to ensure a safe closing of transistors blocking resistors R1-R4 are installed between their gates and emitters. The limitation of power pulses amplitude and protection against a potential breakdown in the gate-emitter transition is ensured by putting suppressors VD1-VD4 parallel to resistors.

To limit the spurious discharge during the commutation of modulator's power keys a limiting snubber has been installed which consists of $\mathrm{R} 6$ and $\mathrm{C} 2$ elements. Another purpose of the snubber is to limit the switch speed of power transistors [6]. The switch speed of transistors has to be limited for the reason that the driver control circuit is based on field complementary transistor structures which in certain conditions may change to an unmanaged state which will lead to a thermal or current breakdown of the driver device.

\section{Conclusion}

A fast-acting complex protection system of a pulse power generator operating on the plasma load has been elaborated. A special attention has been paid to the circuit implementation of protection paths against a potential breakdown and to the quick-action of device.

The application of fast-acting optical systems of data transfer in the composition of the current and thermal protection circuits allows to block the operation of pulse power generator with the lapse of time not exceeding 100 nanoseconds.

The analysis of correlation between the temperature of transistor frames and the value of pulse current flow- ing through them is carried out by a microprocessor control system which allows to correct the threshold of the complex protection system actuation in the process of device operation.

\section{REFERENCES}

[1] D. V. Godun, S. V. Bordusov, A. P. Dostanko. The software-controlled generator of high-voltage impulses for plasma technological application. Acta Technica 56:332-337, 2011.

[2] D. V. Godun, A. P. Dostanko. Control of high-speed IGBT modules in a converter cascade of high-power pulsed inverter. In Modern facilities of communication materials: works of XVI International Scientific and Technical Conference, p. 67. Belarus, Minsk, 2011.

[3] D. V. Godun, A. P. Dostanko, S. V. Bordusov. Stabilization and regulation of value of an output voltage of a high-voltage sources for forming gas-discharge devices with the combined analogue-digital method of transformation. In Proceedings of the report of III International scientific and technical conference "Modern methods and technologies of creation and processing of materials", pp. 233-235. Minsk, Belarus, 2008.

[4] A. Kolpakov. Schematic capture of high-voltage power converters. Power electronics 2:28-32, 2007.

[5] A. Polischuk. Schematic capture of modern high power sources for telecommunications equipment and systems of industrial automation. Power electronics 2(1-4):70-74, 2005.

[6] K. Suker. Power electronics. Developer's Guide. DODEKA-XXI, Moscow, 2008. 